\title{
Iniciación al lenguaje algebraico en aulas multilingües: Contribuciones de un proyecto en desarrollo
}

Núria Planas, Universitat Autònoma de Barcelona (Barcelona)

Recibido el 8 de mayo de 2012; aceptado el 19 de septiembre de 2013

\author{
Iniciación al lenguaje algebraico en aulas multilingües: Contribuciones de un proyecto en \\ desarrollo
}

\section{Resumen}

Se informa sobre un estudio cuyo objetivo es analizar de qué modo aprender matemáticas en una lengua que aún no se domina influye en la construcción del lenguaje algebraico. Los datos proceden de aulas en Barcelona con alumnado latinoamericano. Se presentan resultados sobre dificultades y oportunidades ligadas al aprendizaje matemático en una L2. La principal tesis es que enseñar matemáticas en una lengua distinta a la del alumno tiene repercusiones en el aprendizaje, que no son necesariamente desfavorables ni beneficiosas. Se ilustran dos casos. El primer caso muestra un alumno que usa un razonamiento geométrico para modelizar una expresión algebraica, pero toma un contenido lingüístico para ofrecer una garantía a un argumento erróneo. El segundo caso muestra una alumna que relativiza bien las diferencias entre dos expresiones algebraicas, pero tiene dificultad para dar sentido al lenguaje algebraico a raíz de una traducción imprecisa.

Palabras clave. Momentos de aprendizaje, Lenguaje algebraico, Alumno bilingüe, Datos de aula, Teoría fundamentada

Iniciación à linguagem algebraico em aulas multilingües: contribuições de um projecto em desenvolvimento

\section{Resumo}

Se relata um estudo cujo objetivo é analisar em que medida o fato de a aprendizagem da matemática numa língua que não domina ainda influencia a construção da linguagem algébrica. Os dados são de salas de aula em Barcelona com estudantes latino-americanos. Nós apresentamos resultados sobre dificuldades e oportunidades de aprendizagem matemáticos relacionados a uma L2. A tese principal é que o ensino de matemática em um idioma diferente do que o estudante tem um impacto na aprendizagem, não necessariamente adversa ou benéfica. Dois casos estão ilustrados. Um caso mostra como um aluno usa o raciocínio geométrico para modelar uma expressão algébrica, mas parte de um conteúdo em língua a oferecer uma garantia de um mau argumento. $O$ outro caso mostra como um aluno minimiza as diferenças entre duas expressões algébricas, mas tem dificuldade em fazer sentido da linguagem algébrica na sequência de uma tradução imprecisa.

Palavras chave. Momentos de aprendizagem, Linguagem algebraico, Aluno bilingüe, Dados de aula, Teoria fundamentada

Para citar: Planas, N. (2013). Iniciación al lenguaje algebraico en aulas multilingües: Contribuciones de un proyecto en desarrollo. Avances de Investigación en Educación Matemática, 3, 25-44 
Initiation to the algebraic language in multilingual classrooms: contributions from an ongoing project

\section{Abstract}

The goal of the reported study is to analyze the influence of learning mathematics in a non dominant language on the construction of the algebraic language. Data come from classes in Barcelona with Latin American students. I present results on mathematical difficulties and opportunities related to learning in a L2. The main thesis is that the teaching of mathematics in a language other than the student's has an impact on learning, not necessarily adverse or beneficial. Two cases are illustrated. One case shows how a student uses geometric reasoning to model an algebraic expression, but takes a linguistic content to wrongly provide a guarantee to an argument. The other case shows how well another student downplays the differences between two algebraic expressions, but has difficulty making sense of the algebraic language due to an imprecise translation. theory

Key words. Learning moments, Algebraic language, Bilingual student, Classroom data, Grounded

Iinitiation au langage algébrique dans des salles multilingues: des contributions d'un projet en cours

\section{Résumé}

Cet article rend compte d'une recherche qui vise à analyser certaines influences d'une langue non encore maîtrisée dans l'apprentissage des mathématiques et, plus spécifiquement, la construction du langage algébrique. Les données proviennent d'une expérimentation effectuée avec des élèves d'origine latino-américaine, issus des écoles de langue catalane. Nous présentons des résultats sur les difficultés et les opportunités d'apprentissage des mathématiques en langue seconde. Ces notions sont utiles pour soutenir la thèse principale: si l'enseignement des mathématiques en langue seconde a un impact sur l'apprentissage même des mathématiques, celle-ci n'est ni un avantage, ni un inconvénient. Deux cas sont illustrés. Un cas montre à quel point l'élève utilise un raisonnement géométrique pour modéliser une expression algébrique, qui part toutefois d'un prétexte linguistique pour soutenir un argument erroné. L'autre cas montre combien une élève relativise avantageusement les différences entre deux expressions algébriques, mais pour qui le sens algébrique demeure vague à la suite d'une mauvaise traduction.

Paroles clés. Moments d'apprentissage, Langage algébrique, Élève bilingue, Données de salle, Théorie fondée

\section{Introducción}

Presento resultados en el marco de un estudio llevado a cabo en tres contextos distintos de aula, con datos recogidos en 2007, 2009 y 2010. La investigación en su totalidad no ha finalizado, pero en estos momentos hay un volumen suficiente de información y análisis. El objetivo último es explorar hasta qué punto el hecho de aprender matemáticas en una lengua que aún no se domina influye en la construcción de significados relativos al lenguaje algebraico. Los datos y la parte del análisis elegidos para este artículo atienden al objetivo más concreto de delimitar dificultades en la adquisición del lenguaje algebraico experimentadas por alumnos que se hallan en el proceso de aprendizaje de la lengua de instrucción. Al respecto, he observado momentos de aprendizaje de alumnos latinoamericanos de enseñanza secundaria en Barcelona. Este grupo supone un tercio del 12,5\% de inmigrantes en España (INE, 2012). En Planas y Civil (2009, 2010) y en Planas y Setati (2009) se argumenta el interés por el alumnado inmigrante latinoamericano dado su rendimiento matemático y la percepción generalizada entre muchos profesores de que su situación es distinta a la del alumnado marroquí, paquistaní, senegalés, chino... por hablar una de las lenguas 
oficiales en Cataluña. Desde una perspectiva más amplia, en Civil y Planas (2012) y en Setati y Planas (2012), se señala que el rendimiento de cualquier grupo de alumnado, sea cual sea su condición lingüística, mejoraría si las prácticas de enseñanza de las matemáticas ahondaran en las relaciones entre usos de la lengua y procesos de aprendizaje en el aula.

El artículo se estructura en cuatro partes. Empiezo comentando la experiencia de ser alumno bilingüe en situación de iniciación al lenguaje algebraico. Sigo con un resumen de los principios metodológicos adoptados en el desarrollo de la investigación y, de ahí, paso a ilustrar resultados mediante cuatro episodios de clase. Acabo con reflexiones sobre la necesidad de realizar otras investigaciones complementarias en nuestro ámbito geográfico e institucional. Lograr avances hacia el establecimiento de relaciones entre aprendizaje matemático y diversidad lingüística requiere articular proyectos de largo recorrido cuyo éxito dependerá de la acción coordinada entre investigadores y equipos, nacionales, internacionales y mixtos. Los avances que así se consigan deberán tener implicaciones en términos de progreso en el área y de comprensión de la dimensión social de los procesos de aprendizaje de las matemáticas.

\section{Breve delimitación teórica y literatura previa}

Pimm (1987) fue pionero al señalar dificultades cognitivas en el aprendizaje matemático asociadas al uso de palabras cuyo significado en matemáticas es distinto al significado en la lengua común del aprendiz. Este tipo de polisemia convive con la complejidad social y lingüística dada por la diversidad de lenguas representadas en una gran mayoría de aulas de todo el mundo. El mayor o menor uso combinado de lenguas tiene a menudo que ver con el nivel de conocimiento de cada una de ellas, pero también con el prestigio otorgado a ciertos grupos lingüísticos, latente en las representaciones que la sociedad elabora en torno a las lenguas y sus hablantes (Civil, 2008, 2009). Esto hace que la condición de bilingüe (léase multilingüe cuando convenga) esté sujeta a la influencia de aspectos estructurales y contextuales. Si bien el grado de bilingüismo es difícil de definir en función de la habilidad, el uso, etc., la condición de bilingüe es un rasgo de la identidad ligado a representaciones sociales. En particular, el alumno de matemáticas bilingüe es aquel a quien se le reconoce la capacidad de aprender matemáticas con dos sistemas lingüísticos distintos. Esta caracterización destaca las vivencias desarrolladas en cada lengua por delante del saber sabio lingüístico.

Por otra parte, durante tiempo se creyó que el alumno bilingüe tenía desventajas cognitivas derivadas de una mayor exposición a dificultades de comunicación en la escuela. Cummins (2000) mostró que las desventajas eran sobre todo de tipo social para la mayoría de grupos que se habían estudiado (e.g., los estudiantes mexicanos en Estados Unidos) y que no podían inferirse con base científica otro tipo de desventajas. En relación con el aprendizaje de las matemáticas, Clarkson (2006) y Parvanehnezhad y Clarkson (2008) han aportado datos de estudiantes vietnamitas e iraníes en Australia con un elevado rendimiento en la resolución de problemas. Otros estudios como el de Kazima (2006) en Malawi sobre la comprensión de términos probabilísticos, han documentado el alto rendimiento matemático de alumnos cuya lengua dominante, el chichewa, es muy distinta al inglés, la lengua de la enseñanza en el país. Así pues, no parece sensato concluir sobre la disposición cognitiva del alumno en función de su condición lingüística. En palabras de Setati (2005), el rendimiento matemático debe interpretarse teniendo en cuenta hasta qué punto los modelos educativos y didácticos 
obstaculizan el uso de lenguas no institucionalizadas. De ahí que no sea posible determinar una relación directa entre nivel de rendimiento matemático y grado de conocimiento de la lengua de instrucción sin atender a las circunstancias educativas, sociales y políticas que influyen en cómo dicho rendimiento se valora.

A continuación y dado que los momentos de aprendizaje que analizo giran en torno al lenguaje algebraico, discuto cuestiones relativas al trabajo de este contenido específico. Comento dificultades iniciales en el acceso al lenguaje algebraico y las sitúo junto a dificultades intrínsecas al aprendizaje en una lengua distinta a la propia.

\subsection{Dificultades iniciales en la comprensión del lenguaje algebraico}

Siguiendo a Drouhard y Teppo (2004), la lectura e interpretación de símbolos con uso algebraico es una actividad lingüísticamente compleja cuyo aprendizaje requiere la atención explícita a los procedimientos de conversión entre las lenguas involucradas, además de la capacidad de priorización de la sintaxis por delante de la semántica, o bien la capacidad de relación de un objeto particular con la clase de objetos que denota, entre otros aspectos. Esta complejidad debe interpretarse en el marco más global dado por la dificultad inherente a la formalización y abstracción de conocimiento matemático. Para reducir el impacto de estas dificultades, Drouhard y Teppo recomiendan que el álgebra sea enseñada como objeto que da lugar a un lenguaje potente y específico, y no solo como método de resolución de problemas y de modelización. Esto supone enseñar los símbolos en relación con su articulación semántica y sintáctica, redimensionando la mera función sustitutiva de números u otros objetos. Por otro lado, aunque la naturaleza del lenguaje algebraico lo distingue de cualquier lengua común, desde la perspectiva del aprendizaje, la iniciación al álgebra debe basarse precisamente en el uso y la comprensión de la lengua común. Gran parte de la literatura en este ámbito (Lins \& Kaput, 2004) señala la comprensión profunda de la lengua común como punto de partida de la iniciación escolar al álgebra y su lenguaje.

Si bien todos los alumnos se enfrentan a las dificultades propias de la introducción al lenguaje algebraico, para el caso de aquellos cuya lengua dominante no es la vehicular de la enseñanza surgen dificultades añadidas. Al respecto, hay un estudio clásico sobre la particularidad de aprender a manejar el lenguaje algebraico en un aula bilingüe, el de MacGregor y Price (1999). En el contexto de Estados Unidos, estas autoras investigaron trayectorias de aprendizaje en clases de secundaria donde alumnos de 11 a 14 años se enfrentaban al uso y la traducción de notación algebraica. Algunos de estos alumnos se encontraban además en proceso de aprendizaje del inglés. Se observó que los alumnos con menor conocimiento del inglés tenían más dificultades en el uso semántico y sintáctico del lenguaje algebraico. Más en general, se determinó que el grado de dominio social y académico de la lengua en la que se realizaban las enseñanzas y los aprendizajes tenía impacto directo en el rendimiento matemático, lo cual no es sorprendente. En el terreno de la enseñanza, MacGregor y Price sugirieron trabajar los paralelismos sintácticos entre lenguaje algebraico y lengua común de modo que el aprendizaje de las matemáticas y el del inglés se retroalimentaran. 


\section{Contexto, participantes y métodos}

La cuestión principal de investigación en el contexto del proyecto es la siguiente: ¿Cómo se relacionan los procesos de aprendizaje de la lengua de enseñanza y de las matemáticas? En este artículo y de acuerdo con el análisis completado hasta setiembre de 2012, la cuestión es la siguiente: ¿Cuáles son algunas de las dificultades en la adquisición del lenguaje algebraico experimentadas por alumnos que se hallan en proceso de aprendizaje de la lengua de instrucción? El proyecto se estructura en las etapas 2007-2011 y 2012-2015. La intención de este desglose no es comparar resultados entre etapas, sino disponer de escenarios empíricos distintos surgidos de modificar aspectos sobre las lenguas que se usan en el aula y su frecuencia. Para las dos etapas, las lenguas son castellano (L1) y catalán (L2). En la primera etapa los profesores tienden a usar ambas a pesar de las directrices de la política linguística local, el alumnado latinoamericano tiene un conocimiento básico de la L2 y las aulas son estrictamente bilingües, sin alumnos cuyas lenguas no sean castellano ni catalán. En la segunda etapa se han empezado a seleccionar profesores que declaran no recurrir a la L1, aulas con más de dos lenguas y alumnos de incorporación reciente al sistema escolar, que llevan apenas un año en él y han dejado de asistir hace poco al aula de acogida.

Entre 2007 y 2011 he tomado personalmente datos en tres aulas de tres centros públicos con alumnado de 13 a 14 años. Para cada aula, he asistido a dos sesiones de clase con una secuencia didáctica sobre lenguaje algebraico. Dispongo de seis sesiones grabadas en video, con el registro de la interacción verbal en grupos de trabajo para cada clase durante la realización de tareas del libro, que es el mismo en las tres clases. Para decidir los participantes seguí criterios de selección de profesores a cargo de aulas multilingües con los que hubiera colaborado (ver en Planas \& Civil, 2009, la relación iniciada en 2006 con el Grupo EMAC-Educación Matemática Crítica). Los tres profesores tenían una cantidad distinta de años de experiencia profesional, que iba desde los cinco hasta los dieciocho. Para todos ellos la titulación de base era Matemáticas, por lo que supuse que dominaban los contenidos de la materia que tendrían que enseñar. Atendiendo a cuestiones prácticas solo se grabaron dos grupos de 3 o 4 alumnos por sesión, manteniendo los grupos en las dos sesiones de cada aula. En los seis grupos (i.e., 3 aulas con 2 grupos por aula) hubo alumnos de familia latinoamericana con el catalán como L2. Así llegué al desarrollo de siete casos de alumno (i.e., 6 grupos con un caso por grupo, excepto un grupo con dos casos en 2010), con variedad en cuanto a dominio de la L2, rendimiento académico y edad de llegada a Cataluña.

A pesar de que los grados de dominio de la L2 para los casos de alumno son distintos, en el momento de la investigación ninguno de ellos estaba en situación de exposición primera al catalán ya que esto solo se da en las aulas de acogida, con programas específicos de enseñanza de la L2. Una cuestión problemática son los criterios de selección de alumnos para el desarrollo de casos. Al respecto, se fijó un mínimo conocimiento de la L2 y una mínima implicación en la actividad matemática. En cuanto al rendimiento en matemáticas y teniendo en cuenta la información proporcionada por el profesor del aula, puede decirse que hubo un caso de nivel bajo, tres de nivel medio y tres de nivel alto. A diferencia de lo que a veces se piensa y de lo que se documentó en Planas y Civil (2009, 2010), el rendimiento académico de estos alumnos (al menos de los que fueron objeto de grabación) no era más bajo que el del resto de alumnos del aula ordinaria. Por otra parte, aunque no siempre el tiempo de 
estancia en un lugar es el mejor indicador del conocimiento de la L2 ya que puede haber un efecto neutralizador por el uso persistente de la L1, se prestó atención a la edad de llegada a Cataluña. Los alumnos de los casos llegaron entre los 6 y los 9 años.

Determinar la temática de enseñanza fue complejo. Quise que hubiera una misma temática de referencia en todas las sesiones de clase, sin tener claro si alguna en particular contribuiría a visibilizar mejor las supuestas relaciones entre lenguas de uso y aprendizaje matemático. Una conversación con M. Setati a inicios de 2007 me llevó a priorizar el lenguaje algebraico como objeto de enseñanza y aprendizaje. En un trabajo en el contexto sudafricano no publicado, Setati había comprobado que profesores y alumnos valoraban el lenguaje algebraico como un mediador de la comunicación matemática en entornos multilingües, y a su vez que el rendimiento en esta temática era bajo entre aquellos alumnos con un escaso dominio académico del inglés, la lengua dominante en la enseñanza. Poco después, consensué con profesores del Grupo EMAC una misma secuencia de enseñanza, con la dedicación de dos sesiones a la introducción del lenguaje algebraico y a su relación con la lengua común. Se consideró el marco didáctico del álgebra como lenguaje, con atención a las dificultades vinculadas con el paso del lenguaje algebraico al lenguaje ordinario y viceversa (ver, por ejemplo, los trabajos de Palarea, 1999, y Socas, 2008, sobre dificultades en la adquisición del lenguaje algebraico). Pedí que ambas sesiones tuvieran trabajo individual en torno a una tarea escrita, trabajo en grupos para la discusión conjunta y puesta en común con el profesor, con tiempos variables en función de la tarea y de la implicación en ella. La Figura 1 muestra parte de la tarea, en la L2, del segundo día de clase.

Expressa en llenguatge algebraic els següents enunciats [Expresa en lenguaje algebraico los siguientes enunciados]:

i) El triple d'un nombre [El triple de un número]

ii) Un nombre de tres xifres [Un número de tres cifras]

(iii) La suma de dos nombres consecutius [La suma de dos números consecutivos]

(iv) Dues vegades la suma de dos nombres [Dos veces la suma de dos números]

Ara fes el camí invers i escriu amb paraules els següents enunciats [Ahora haz el camino inverso y escribe con palabras los siguientes enunciados]:
a) $2 x$
c) $4 x^{2}$
b) $2 x+1$
d) $5 x y$

Torna a usar el llenguatge algebraic per expressar les següents relacions [Vuelve a usar el lenguaje algebraico para expresar las siguientes relaciones]:

1) Relació entre àrea, alçada i base d'un triangle [Relación entre área, altura y base de un triángulo]

2) Relació entre perímetre i radi d'una circumferència [Relación entre perímetro y radio de una circunferencia]

3) Relació entre aresta i volum d'un cub [Relación entre arista y volumen de un cubo]

4) Relació entre hipotenusa i catets d'un triangle rectangle [Relación entre hipotenusa y catetos de un triángulo rectángulo]

Figura 1. Fragmento de la segunda tarea de clase (con traducción incorporada para el artículo) 
Para el análisis del trabajo en grupos previo a la puesta en común, he examinado: i) los objetos matemáticos en proceso de discusión -¿de qué matemáticas se habla?, ii) las interpretaciones en torno a dichos objetos - ¿sobre qué significados matemáticos hay (des)acuerdo?, iii) los cambios efectivos de lengua - ¿cuándo se va de la L1 a la L2 y viceversa?, iv) las referencias explícitas al uso de la L1 y/o la L2 -¿cómo se explican los cambios? Son cuatro aspectos relativos a las dos dimensiones principales de análisis, el contenido matemático y el uso de las lenguas. Cada aspecto se describe por separado como preámbulo de la búsqueda de relaciones entre ambas dimensiones. Para cada grupo y sesión, por tanto, el análisis empieza con la identificación del contenido matemático de la interacción y de los usos de las lenguas por parte de los participantes involucrados. Esta parte del análisis es esencialmente descriptiva. Está pensada para agilizar la posterior interpretación de relaciones entre lo matemático y lo lingüístico.

Tras recopilar datos sobre contenido matemático y uso de lenguas en situaciones de interacción en grupo, se llega al proceso inductivo de conceptualización de relaciones cualitativas. Este es ya un paso interpretativo de transformación de datos descriptivos para el cual se toman métodos basados en la Teoría Fundamentada (Glaser, 1969). La aplicación sistemática de estos métodos ha venido facilitada por el uso de un programa informático de organización de datos y la ayuda de un técnico de apoyo experto en la manipulación del programa. En el entorno informático se ha establecido un doble sistema de códigos para contenido matemático y uso de lenguas, que ha sido modificado en varios momentos del proceso. A pesar de estar lejos de la construcción de una teoría formal, la explicación de relaciones emergentes en la sección de resultados es prometedora. La inclusión de datos proporcionados por la segunda etapa del proyecto tiene que llevar a consolidar algunas de estas relaciones, lo cual a su vez tiene que permitir la elaboración de temas que integren aprendizaje matemático y uso de lenguas.

La Tabla 1 ilustra parte del doble sistema de códigos que ha servido para organizar datos. Los códigos sintetizan interpretaciones sugeridas por alguno(s) de los siete alumnos en interacción matemática con su grupo. He marcado con un asterisco (*) los códigos ejemplificados en la sección de resultados. Como se verá, no hay una distinción individualizada de resultados en relación con los códigos de uno u otro tipo. Aunque desde la investigación en educación matemática podría pensarse positivo separar los resultados que se refieren al uso de la L1 y la L2 de aquellos que se refieren al aprendizaje del contenido matemático, tanto la naturaleza de los datos como el posicionamiento adoptado hacen poco recomendable intentar una distinción en este sentido. Por ejemplo, en 'De la falta de vocabulario a la interpretación geométrica' (ver primer apartado de la sección de resultados), hay una oportunidad de aprendizaje cuando uno de los alumnos "lee" geométricamente una expresión algebraica. A raíz del análisis de los datos de clase, defiendo que tal oportunidad viene facilitada por la falta de vocabulario del alumno en la L2. Por ello argumento la imposibilidad de desligar aspectos matemáticos de aspectos lingüísticos en la generación e interpretación de resultados. Este es un argumento para el desarrollo de estudios sobre el aprendizaje del lenguaje algebraico en aulas multilingües, no necesariamente aplicable a estudios sobre el aprendizaje del lenguaje algebraico en general.

Antes de presentar resultados, conviene explicar el contenido de la Tabla 1 . He optado por no tomar códigos primarios teóricos y elaborar directamente códigos que representen agrupaciones de momentos de aprendizaje con algún aspecto común (las agrupaciones se han transcrito después de la creación del código correspondiente). Los dos sistemas surgen de identificar significados matemáticos y usos lingüísticos en la 
interacción entre alumnos dentro del grupo de trabajo, a lo largo de un proceso continuo de ida y vuelta entre datos y códigos. Esto ha llevado a una gran diversidad de códigos sin conexión aparente que atienden a aspectos distintos de la interacción. Ha sido el conocimiento generado por la acumulación de datos y códigos, con la revisión constante de unos y otros, lo que ha inspirado la inferencia de relaciones. En este sentido, los dos conjuntos de códigos se han ido reduciendo progresivamente hasta solo incluir de forma ordenada los más frecuentes. Las columnas de la Tabla 1 incluyen los ocho primeros más frecuentes en fecha setiembre de 2012. Tal reducción ha permitido vislumbrar regularidades de permanencia de códigos y de relaciones inter e intra sistemas. La obtención de resultados se ha consolidado durante la construcción de temas iniciales que integran códigos matemáticos y linguiísticos. No se ha llegado al punto de saturación teórica, pero sí se ha conseguido afinar una codificación más selectiva que abierta. Por otra parte, es de esperar que muchos de los actuales códigos y temas vuelvan a ser objeto de desarrollo analítico en la segunda etapa del proyecto.

Tabla 1. Fragmento del doble sistema de códigos.

\begin{tabular}{|l|l|}
\hline \multicolumn{2}{|c|}{ Doble sistema de codificación } \\
\hline \multicolumn{1}{|c|}{ Códigos sobre contenido matemático } & \multicolumn{1}{c|}{ Códigos sobre uso de L1 y L2 } \\
\hline $\begin{array}{l}\text { Interpretación geométrica*: Representaciones } \\
\text { físicas de relaciones en una expresión } \\
\text { algebraica }\end{array}$ & $\begin{array}{l}\text { Alternancia regular*: Uso fluido de L1 y L2 } \\
\text { según el interlocutor, la situación de habla, } \\
\text { etc. }\end{array}$ \\
\hline $\begin{array}{l}\text { Distinción de fórmulas particulares*: } \\
\text { Vínculos entre expresión algebraica, fórmula } \\
\text { y propiedad }\end{array}$ & $\begin{array}{l}\text { Creación de palabras*: Uso de la L2 } \\
\text { mediante la invención de palabras con } \\
\text { similitud en la L1 }\end{array}$ \\
\hline $\begin{array}{l}\text { Diversidad de representaciones*: Asociación } \\
\text { de varias expresiones para indicar casos } \\
\text { numéricos }\end{array}$ & $\begin{array}{l}\text { Variedad interna de la lengua*: Distinción } \\
\text { de modismos y palabras dentro de la L1/L2 }\end{array}$ \\
\hline $\begin{array}{l}\text { Uso automático del álgebra*: Conversión de } \\
\text { enunciados al lenguaje algebraico sin dar } \\
\text { sentido }\end{array}$ & $\begin{array}{l}\text { Traducción imprecisa*: Discordancia entre } \\
\text { significados del término original y su } \\
\text { traducción }\end{array}$ \\
\hline $\begin{array}{l}\text { Organización de paréntesis: Evaluación de } \\
\text { niveles de prioridad de operaciones }\end{array}$ & $\begin{array}{l}\text { Cambio puntual a la L2: Uso de la L2 para } \\
\text { referenciar o preguntar un término técnico }\end{array}$ \\
\hline $\begin{array}{l}\text { Marcas aritméticas y algebraicas: Discusión } \\
\text { sobre la mayor presencia de uno u otro } \\
\text { lenguaje }\end{array}$ & $\begin{array}{l}\text { Síntesis en la L1: Resumen en la L1 de un } \\
\text { texto presentado de modo exclusivo en la } \\
\text { L2 }\end{array}$ \\
\hline $\begin{array}{l}\text { Signos invisibles de operación: Comparación } \\
\text { entre signos de suma, resta y multiplicación }\end{array}$ & $\begin{array}{l}\text { Abandono de la alternancia: Uso mantenido } \\
\text { de la L1/L2 hasta concluir un razonamiento }\end{array}$ \\
\hline $\begin{array}{l}\text { Condición de incógnita: Asimilación de la } \\
\text { incógnita con letras en la expresión } \\
\text { algebraica }\end{array}$ & $\begin{array}{l}\text { Reestructuración del mensaje: Explicación } \\
\text { de un texto previo en la L1/L2 mediante la } \\
\text { L2/L1 }\end{array}$ \\
\hline
\end{tabular}

\section{Discusión de resultados sobre oportunidades y dificultades}

En esta sección introduzco cuatro resultados, dos centrados en dificultades relativas al aprendizaje de las matemáticas en una lengua que el alumno bilingüe aún no domina y otros dos centrados en oportunidades de aprendizaje derivadas de esta misma situación. Tomo cuatro momentos de clase aunque cada resultado se fundamenta en la agrupación de varios momentos. Asimismo, la presentación conjunta y equilibrada de dificultades y oportunidades responde a la principal tesis del artículo y de mi trayectoria de investigación en este ámbito: enseñar matemáticas en una lengua que el alumno no domina lo bastante tiene repercusiones en el aprendizaje, que no son 
necesariamente desfavorables ni beneficiosas. Lejos de ser contradictorios, los resultados apuntan a la complejidad de los entornos bilingües de enseñanza y aprendizaje donde alumnos que solo utilizan su L2 en horario escolar consiguen progresar académicamente.

De acuerdo con Clarkson (2006), Kazima (2006) y Parvanehnezhad y Clarkson (2008), las oportunidades indican que el alumno bilingüe que aprende matemáticas en su L2 puede mantener un elevado rendimiento en la materia, superior incluso al de alumnos que aprenden en su L1. Los resultados del actual estudio señalan que estos mismos alumnos pueden experimentar dificultades en ciertos momentos de acceso al lenguaje algebraico. Para el uso coordinado de la L1, la L2 y el lenguaje algebraico, se requiere una sofisticada comprensión de las relaciones posibles entre sistemas lingüísticos, las cuales no son triviales sin una enseñanza específica. En lo que sigue, ilustro los resultados mediante dos casos de alumnos nacidos en Ecuador, Roberto y Amelia de 13 años, que forman parte de dos grupos en dos de las aulas y que llevaban poco más de un año en Barcelona en 2009 y en 2010 respectivamente. Roberto y Amelia son descritos por sus profesores de matemáticas como alumnos "con un buen rendimiento en clase". Los dos primeros resultados ponen de relieve cómo Roberto introduce con éxito un razonamiento geométrico para modelizar una expresión algebraica, pero recurre a su L1 para ofrecer una garantía a un argumento erróneo. Los dos últimos resultados ponen de relieve cómo Amelia amplía las representaciones posibles para casos de números, pero más tarde tiene dificultad para dar sentido al uso del lenguaje algebraico debido a una traducción imprecisa que se le ha proporcionado.

En las transcripciones reproduzco la lengua utilizada por los alumnos. Aunque la proximidad entre catalán y castellano permitiría no traducir los textos, esta opción debilitaría la tesis sobre la distancia lingüística que los alumnos latinoamericanos experimentan en las aulas catalanas. Cataluña es una comunidad bilingüe donde la mayoría de la población crece hablando catalán y castellano con fluidez ya que ambas lenguas son frecuentes en los medios de comunicación, en los lugares de trabajo y, más en general, en la calle. No obstante, la situación del alumnado latinoamericano es distinta al no haber sido expuesto con similar intensidad a las dos lenguas desde edades tempranas. En cierto modo, un lector internacional con dominio del castellano podría experimentar esta misma distancia lingüística si no está familiarizado con el catalán.

\subsection{Roberto: Oportunidades y dificultades matemáticas ligadas a la lengua}

La falta de conocimiento de vocabulario matemático en la L2 aparece a menudo en los datos de las tres aulas. Lo que debe considerarse un resultado novedoso es que a raíz de ello surjan oportunidades de aprendizaje relativas al lenguaje algebraico. En el siguiente fragmento, Roberto no recuerda la palabra catalana para número impar y a raíz de ello elabora un razonamiento geométrico para interpretar la expresión $2 x+1$ (ver Tabla 1). Los compañeros de grupo son Joana y Miquel, ambos con el catalán como L1.

Oportunidad-De la falta de vocabulario a la interpretación geométrica

Roberto: ¿Tú que escribiste para este otro $\{2 x+1\}$ ?

Joana: Un número impar.

Roberto: ¿Pero qué escribiste en la hoja?

Joana: Esto, \{lee\} un nombre senar [un número impar]. 
Roberto: Senar [Impar]... no me acordaba.

Miquel: Jo també he posat nombre senar. Tu què has posat? [Yo también he puesto número impar. ¿Tú que has puesto?]

Roberto: Rectángulo de... costat dos i quadrat de costat $u$ [lado dos y cuadrado de lado uno].

Miquel: A veure, com? [A ver, ¿cómo?]

Roberto: Es que no me acordaba de senar [impar].

Miquel: Pues haber puesto imparell [impar].

Roberto: No sé, me sonaba mal.

Joana: Però és bona idea... És com si fossin dues àrees [Pero es buena idea... Es como si fueran dos áreas].

Roberto: És que són dues àrees! [¡Es que son dos áreas!].

Miquel: Et sonava malament? [¿Te sonaba mal?]

Joana: Si li sona bé, no ho fa [Si le suena bien, no lo hace].

Roberto: ¡Chévere! Miro las áreas del dos equis y del uno.

La expresión $2 \mathrm{x}+1$ viene precedida de la discusión en torno a 2x, que Roberto, Joana y Miquel asocian con el concepto de número par sin elaborar un razonamiento geométrico complementario donde $2 \mathrm{x}$ represente el área de un rectángulo con dos unidades de base, o bien donde $2 x$ represente una función, entre otras posibilidades. Tras la dificultad por recordar la palabra para número impar en la L2, Roberto llega a un razonamiento geométrico para $2 x+1$. Este comportamiento matemático se condensa en el código 'Interpretación geométrica' (ver Tabla 1), sobre la representación física de relaciones dadas por una expresión algebraica (en Socas, Camacho, Palarea, \& Hernández, 1999, se pueden consultar procesos de descubrimiento de estructuras geométricas dadas por expresiones algebraicas). Además de la interpretación geométrica de la expresión algebraica, hay una argumentación correcta cuando el alumno justifica que los dos términos de la suma se refieren a un rectángulo y un cuadrado. El pensamiento algebraico y el geométrico son de carácter sustancialmente distinto con influencia de múltiples variables. No debe, por tanto, suponerse que la falta de vocabulario por si sola tiene un efecto determinante en Roberto, aunque sí es razonable concluir que actúa de mediador. A pesar de que la tarea y la organización curricular del profesor contribuyen a establecer límites entre álgebra y geometría, Roberto integra el significado aritmético para el caso especial de número impar con el geométrico.

En la interacción, Roberto combina la L1 y la L2 en un comportamiento de 'Alternancia regular' (ver Tabla 1), según el interlocutor, la situación de habla u otros factores interrelacionados. La alternancia se produce a nivel de la frase, como se ve en varios turnos, o bien a nivel de la conversación, como se ve en la totalidad de la transcripción que no reproduzco. Atendiendo a otros momentos de aprendizaje, el grupo de estos alumnos aparece como contexto donde es posible el uso flexible de las dos lenguas. Asimismo, los distintos momentos con alternancia de lenguas sugieren una tendencia al uso de la L1 en la elaboración de razonamientos conceptuales (e.g., "Miro las áreas del dos equis y del uno"), que contrasta con el uso combinado de la L1 y la L2 en razonamientos más rutinarios y/o procedimentales (e.g., "Vas llegint terme a terme $i$ escrivint”, extraído de una interacción que no reproduzco). Aunque es plausible esperar tal resultado si atendemos a lo encontrado en aulas sudafricanas por 
Setati (2005), quien probó el mayor uso de la L1 en actividades no rutinarias de razonamiento heurístico, este es un resultado que tendrá que ser revisado en la segunda etapa del proyecto.

Sigue un momento de aprendizaje de la misma sesión donde Roberto experimenta una dificultad matemática. En el grupo, los tres alumnos han pensado por separado cómo expresar con lenguaje algebraico el Teorema de Pitágoras, enunciado como la relación entre hipotenusa y catetos de un triángulo rectángulo (ver Tabla 1).

Dificultad-De un juego con palabras a la idea de fórmula concreta

Roberto: ¿Por qué me lo has corregido? $\left\{\mathrm{h}^{2}=\mathrm{b}^{2}+\mathrm{c}^{2}\right.$, en lugar de $\left.\mathrm{h}^{2}=\mathrm{c}^{2}+\mathrm{c}^{2}\right\}$

Joana: Perquè estigui bé [Para que esté bien].

Roberto: Pero... la fórmula és així [la fórmula es así].

Joana: I què? [¿Y qué?]

Roberto: No lo cambio.

Joana: Ho tindràs malament [Lo tendrás mal].

Roberto: Los dos catetos son catets [catetos], ¿no? ¿O uno se llama batet [bateto] y otro catet [cateto]?

Miquel: Vinga! Catet [iVa! Cateto].

Joana: Pots posar una ix però llavors has de posar una y. No sempre valen el mateix [Puedes poner una equis pero entonces tienes que poner una y. No siempre valen lo mismo].

Roberto: Són diferents, però són catets, sí? [Son diferentes, pero son catetos, ¿sí?] Lo dejaré claro cuando sepa el triángulo que tengo. Es una fórmula, pero concreta.

Joana: Ho has de deixar clar des de ja perquè és una fórmula general [Lo tienes que dejar claro desde ya porque es una fórmula general].

Este fragmento ilustra el código 'Distinción de fórmulas particulares' (ver Tabla 1), que agrupa momentos de aprendizaje en los cuales se vincula, implícita o explícitamente, una o varias expresiones algebraicas con las nociones de fórmula, fórmula general y propiedad. Desde la perspectiva de la comprensión del lenguaje algebraico, este ejemplo informa sobre la confusión de Roberto, quien no tiene inconveniente en usar la misma letra para representar algebraicamente dos valores numéricos que pueden ser distintos (en Font \& Contreras, 2008, se puede leer una discusión sobre los objetos matemáticos con representaciones que admiten ser interpretadas simultáneamente como particulares y generales). Es probable que Roberto esté familiarizado con una expresión algebraica mal formulada del Teorema de Pitágoras, que incluso le puede haber sido presentada en el contexto escolar. Por otra parte, resulta interesante la diferenciación implícita sugerida entre fórmula general y fórmula concreta, según si se enuncia una propiedad que se cumple para todos los casos de triángulos rectángulos o bien una propiedad que no se cumple siempre. El uso del término fórmula concreta sugiere la existencia de fórmulas particulares extraíbles de la primera. Cuando se menciona la idea de casos particulares de triángulos (i.e., "Lo dejaré claro cuando sepa el triángulo que tengo"), se están de algún modo delimitando dos fórmulas particulares según si los dos catetos miden igual (i.e., $h^{2}=c^{2}+c^{2}$ ) o tienen distinta medida (i.e., $\mathrm{h}^{2}=\mathrm{b}^{2}+\mathrm{c}^{2}$ ).

$\mathrm{El}$ anterior ejemplo forma parte de los momentos de aprendizaje agrupados dentro del código 'Creación de palabras' (ver Tabla 1), sobre el uso de la L2 mediante la 
invención de palabras con similitud en la L1. Roberto se basa en una invención de este tipo (i.e., batet) para argumentar la idoneidad de la expresión $\mathrm{h}^{2}=\mathrm{c}^{2}+\mathrm{c}^{2}$ al vincular la doble selección de la letra $c$ con la primera letra de la palabra que sustituye. La cuestión de fondo es que la palabra cateto hace referencia a dos objetos matemáticos distintos. Los dos catetos de un triángulo rectángulo son equivalentes si consideramos que los dos son menores que la hipotenusa, o bien que los dos conforman el ángulo recto. No obstante, no son el mismo objeto geométrico aún cuando tienen la misma longitud. La no distinción entre los dos catetos en la lengua común hace la simbolización algebraica de los dos catetos costosa. Mediante un juego de palabras, Roberto se asegura que esto ocurra para la L2 (i.e., catet, no batet) y la L1 (i.e., cateto). En este sentido, el alumno es capaz de señalar la coincidencia entre la L1 y la L2 con respecto a la letra de inicio de la palabra sustituida. Lo significativo es que Roberto usa datos sobre la L2 para ofrecer una garantía en la que sostener su argumento erróneo. La L2 interviene como elemento y contenido en la elaboración de un razonamiento que pretende ser matemático. Hay más momentos de aprendizaje en los cuales Roberto se refiere al conocimiento de su L2 para dar una apariencia de argumentación a inferencias matemáticamente erróneas.

Cuando se refiere a la relevancia del lenguaje, Wittgenstein (1953) explica que los usos de las palabras y los juegos de significados entre ellas no son solo recursos lingüísticos sino también formas de construir conocimiento sobre la realidad. Esto es lo que le ocurre precisamente a Roberto. Este alumno utiliza la palabra catet(o) para validar la fórmula $h^{2}=c^{2}+c^{2}$, del mismo modo que hubiera utilizado la palabra inventada batet(o) para validar la fórmula $\mathrm{h}^{2}=\mathrm{b}^{2}+\mathrm{c}^{2}$ si Miquel y Joana hubieran aceptado este juego del lenguaje. La posibilidad de jugar simultáneamente con la L1, la L2 y el lenguaje algebraico conduce a una dificultad debido a que Roberto asemeja la palabra, ya sea en la L1 o en la L2, con la validez de la fórmula. Se está simplificando la naturaleza del lenguaje algebraico mediante la atención exclusiva a las palabras, sin incorporar los significados de las palabras en relación con los objetos matemáticos.

\subsection{Amelia: Oportunidades y dificultades matemáticas ligadas a la lengua}

En el siguiente momento de aprendizaje, la conversación empieza cuando una alumna, Amelia, pregunta sobre la conversión al álgebra de la expresión "un número de tres cifras" (ver Figura 1). La propuesta de Amelia, xyz, tiene en cuenta la representación del valor posicional de las cifras habitual en teoría de números, a diferencia de la propuesta de sus compañeros, $100 x+10 y+z$, más propia de las convenciones algebraicas.

Oportunidad-De la variedad lingüística a las representaciones de números

Amelia: ¿Por qué has puesto esto $\{100 \mathrm{x}+10 \mathrm{y}+\mathrm{z}\}$ ?

Eduard: Porque si fueran dos cifras pondría \{escribe $\} 10 \mathrm{y}+\mathrm{z}$.

Amelia: Pero, ¿por qué lo complicas?

Eduard: Què proposes? [¿Qué propones?]

Amelia: Pues solo \{escribe $\}$ xyz. Y si es de dos, \{escribe $\}$ yz.

Eduard: No està bé [No está bien].

Amelia: ¿Por qué?

Silvia: Tu poses com si multipliquessin [Tú pones como si multiplicaran]. 
Eduard: I són centenes, desenes i unitats [Y son centenas, decenas y unidades].

Amelia: La suma de les centenes, les desenes... [La suma de las centenes, las decenas...] y las unidades.

Eduard: No, perquè hi ha el cent i el deu [No porque hay el cien y el diez].

Amelia: Pero si equis es cero no hay el cien, y a lo mejor tampoco hay el diez. De quiteña cambiaba diez barras de madera por una. Con cero barras ese lugar no está... y es como lo pongo yo.

Eduard: A veure, torna-ho a explicar [A ver, vuélvelo a explicar].

Amelia: Lo que digo es que son casi lo mismo.

Silvia: $\quad$ El que tu dius seria molt diferent! [iLo que tú dices sería muy diferente!]

Amelia: No son iguales como un medio y dos cuartos, pero son bastante lo mismo.

Silvia: Doncs explica-ho perquè jo no ho veig [Pues explícalo porque yo no lo veo].

La transcripción puede hacer pensar que Amelia no entiende el uso algebraico de los símbolos para representar expresiones aritméticas de forma genérica. La modelización de un número de tres cifras con la expresión $x y z$ podría indicar una dificultad en este sentido, pero también podría considerarse que la alumna se resiste a sustituir la representación habitual de números que le ha sido enseñada hasta su inicio al álgebra (en Socas, 2002, se discute la ambigüedad intrínseca de los sistemas de representación decimal). Visto así, este fragmento ilustra una oportunidad de aprendizaje, codificada como 'Diversidad de representaciones' (ver Tabla 1). Una segunda oportunidad surge con la comparación entre $100 x+10 y+z$ y $x+y+z$ para el valor numérico cero de $x$ e $y$. Las dos expresiones algebraicas no son equivalentes porque no tienen el mismo valor numérico para cualquier valor que demos a las letras, aunque son "bastante lo mismo", tal como Amelia sugiere, por ser válidas para el "número de tres cifras con cero centenas y cero decenas". Mediante la particularización, se contribuye a que Eduard y Silvia atiendan a las similitudes entre expresiones algebraicas no equivalentes. Asimismo, Amelia compara la relación de equivalencia entre dos representantes de la clase del número racional 0.5 ("un medio y dos cuartos") y la relación de igualdad entre dos expresiones algebraicas para un valor numérico.

Amelia usa mayoritariamente su L1 y se refiere a un término ajeno al castellano peninsular, el de "quiteña", modismo para habitante de la ciudad de Quito. Así como hay una variedad lingüística de castellano hablada en los territorios en contacto con el catalán, hay una variedad lingüística de castellano hablada en Ecuador. Este apunte es esencial para relativizar la presencia de dos únicas lenguas comunes en las aulas del estudio. En el caso de Roberto, el modismo "chévere" no afecta la fluidez en la comunicación. Sin embargo, en el caso de Amelia, el término quiteña viene a complicar la comprensión de una frase que de por sí ya resulta oscura, en relación con las barras de madera. Esta alumna habla de los bloques multibase, un material habitual en las escuelas de primaria para el trabajo de la estructura del sistema de numeración decimal; no obstante, lo hace de un modo difícil de comprender, por suponer un cambio de contenido en la conversación y por diferir su L1 de la L2 de Silvia y Eduard. Amelia recurre a la representación física del número de tres cifras e imagina la agrupación de diez unidades de un orden pasando a una unidad de orden superior. La oportunidad de aprendizaje sigue latente sobre todo debido a las reacciones de Eduard y Silvia, pero hay un obstáculo de tipo lingüístico que actúa de mediador y condiciona el alcance de la oportunidad. El código 'Variedad interna de la lengua' (ver Tabla 1) 
indica el uso de modismos y palabras distintas con mismo significado dentro de una lengua.

Paso a resumir un momento de aprendizaje de la misma sesión. Amelia experimenta una dificultad que no se acaba convirtiendo en oportunidad matemática, sino más bien en una circunstancia desfavorable para la construcción comprensiva de la noción de lenguaje algebraico. Los tres alumnos están pensando cómo expresar algebraicamente la relación entre arista y volumen de un cubo (ver Figura 1).

\section{Dificultad-De una traducción imprecisa al álgebra sin significado}

Amelia: ¿Qué quiere decir aresta [arista]?

Eduard: Lado.

Amelia: ¿Para un cubo el lado se dice aresta [arista]?

Eduard: Lado y ... aresta són el mateix [arista son lo mismo].

Silvia: Ella et pregunta si només són el mateix per un cub [Ella te pregunta si solo son lo mismo para un cubo].

Eduard: Jo què sé! [iYo qué sé!]

Amelia: Yo nunca he dicho aresta [arista] de un cuadrado.

Eduard: Pues lo puedes decir.

Silvia: No la liïs, ha de ser l'aresta d'un cub, d'un prisma, d'un con... [No la líes, tiene que ser la arista de un cubo, de un prisma, de un cono...]

Amelia: ¿Cuál es el lado de un cono?

Silvia: L'aresta? [¿La arista?]

Amelia: ¿Cuál es la aresta [arista]?

Silvia: Quin problema tens? [¿Qué problema tienes?]

Amelia: ¿Los lados redondos valen? Bueno, las arestes [aristas] redondas...

Silvia: Mira, es igual, así no acabaremos nunca. Esto es \{escribe $a^{3}$ porque es el volumen. No ens estan demanant que donem tantes voltes [No nos están pidiendo que demos tantas vueltas].

Amelia: ¿Pasamos de lo quiere decir y vamos directos al álgebra? Vale.

En esta transcripción, la dificultad matemática primera tiene que ver con la confusión entre elementos de figuras planas y de cuerpos sólidos. La comparación entre cuadrado y cubo deriva hacia la comparación entre cuerpos con caras planas y cuerpos con caras curvilíneas. Aquí, aparece una segunda dificultad ligada a la intersección geométrica entre caras no planas y líneas curvas. Efectivamente las aristas son lados, pero son los lados de las caras del cuerpo sólido. Cuando Eduard iguala semánticamente aresta y lado, no clarifica este aspecto: la arista es del cuerpo y el lado es de una cara del cuerpo. Una distinción al respecto hubiera ayudado a visualizar el lado de un cono por medio de imaginar el lado de la base del cono, que es el perímetro de la circunferencia. Este perímetro es la única arista del cono al ser la línea fronteriza entre las dos caras. El fragmento acaba con un salto repentino hacia el uso de lenguaje algebraico sin haber solucionado las dudas sobre conocimiento geométrico (en Filloy, Puig, \& Rojano, 2008, se reportan ejemplos sobre la manipulación del álgebra sin sentido y sobre la asignación de significados erróneos previos al proceso de simbolización). Al aceptar la manipulación sin sentido del lenguaje algebraico, se 
prepara un 'Uso automático del álgebra' (ver Tabla 1). Para comprender el sentido de este lenguaje se requiere interiorizar la doble relación entre objetos matemáticos (e.g., arista de un cubo y volumen del cubo) y expresiones algebraicas asociadas (e.g., $a, a^{3}$ ), lo cual no es posible sin una comprensión previa del significado de los objetos involucrados.

De nuevo, estamos ante un momento de aprendizaje donde se usa de forma combinada la L1 y la L2. Desde la perspectiva de la lengua en interacción con las matemáticas, lo interesante es la innecesaria ambigüedad de la traducción de aresta como lado, así como su impacto en la conversación. Para este término hay una traducción precisa, arista, que Eduard probablemente conoce. Sin embargo, la confusión matemática entre arista y lado pueden haber llevado a proporcionar una palabra con significado no equivalente. Este fenómeno, que ocurre en varios momentos de aprendizaje con otros términos matemáticos, se corresponde con el código 'Traducción imprecisa' (ver Tabla 1). Por un lado, tenemos la polisemia derivada del lado entendido como lado de una figura o como lado de la cara de un cuerpo y, por otro, la falsa sinonimia derivada de la traducción de aresta por lado. Estos inconvenientes, intrínsecos a la dificultad de cualquier traducción técnica, generan una situación propicia para que el lenguaje algebraico se acabe viendo como un recurso orientado a manipular sin sentido un enunciado matemático. En la mayoría de ocasiones donde aparece este código, una explicación extensiva del término, en lugar de una traducción, habría supuesto una opción menos arriesgada en la interacción matemática.

Cuando Amelia pide saber qué quiere decir aresta, activa una estrategia de enseñanza por parte de Eduard basada en una traducción del término. No obstante, la traducción literal (cuando es posible) no debe confundirse con la enseñanza del término, que requiere una explicación extensiva. Kazima (2006) ha señalado riesgos asociados a traducciones imprecisas en situaciones de uso flexible de dos lenguas en el aula de matemáticas. El uso flexible de lenguas va más allá de las estrategias puntuales de traducción de palabras y frases. Se requieren, en particular, estrategias de parafraseo que den cuenta de los contenidos matemáticos de un discurso. El hecho de reelaborar un enunciado matemático en la misma lengua en el cual se ha expresado, o bien en otra distinta, es en sí mismo un proceso de reconstrucción matemática. Esta estrategia es común en las aulas monolingües, donde "profesores y alumnos hacen uso de parafraseos al hablar sobre los contenidos de enseñanza y aprendizaje, tratando de reformular con palabras distintas lo que ya se ha dicho e incluyendo así significados nuevos" (Goizueta \& Planas, 2013).

\section{Consideraciones finales y prospectiva}

Las evidencias obtenidas en la primera fase del proyecto señalan que no hay por qué presuponer un menor rendimiento matemático para alumnos instruidos en la L2. Para contenidos específicos vinculados con la enseñanza y el aprendizaje del álgebra, se han proporcionado tanto oportunidades como dificultades de aprendizaje. Lo que desde mi posicionamiento social es cierto para cualquier grupo de alumnos (no hay que presuponer a priori un menor rendimiento matemático en comparación con otros grupos), debe ponerse de relieve con especial intensidad para aquellos grupos a los cuales se les suponen escasas posibilidades de promoción académica. Por otro lado, se han mostrado ejemplos de implicación en la actividad matemática donde la lengua se ha hecho presente de distintos modos y ha resultado ser un factor mediador en la 
relación del alumno con la interpretación del lenguaje algebraico. Al resumir el equilibrio entre oportunidades y dificultades surgido del análisis del conjunto de datos, aporto un panorama del complejo papel que cumple la diversidad de lenguas en el aprendizaje del lenguaje algebraico. Es un papel complejo en primer lugar por el peso que los propios alumnos otorgan a la comprensión en la L2, pero además a causa de las dependencias existentes entre la conversión de lenguas donde una de ellas es algebraica.

La conclusión anterior debe ubicarse dentro de una reflexión más general con implicaciones para la formación del profesorado y los políticos con responsabilidades. Cualquier consideración sobre el uso flexible de las lenguas en un aula debe ir acompañada de una declaración de principios sobre el derecho de todos los alumnos a aprender la(s) lengua(s) del país de acogida, y a usar su L1 con fines de aprendizaje sin ser penalizados por ello. Estas ideas sobre el uso flexible de las lenguas han sido reforzadas en la Conferencia del 21 Estudio ICMI sobre Educación Matemática y Diversidad Lingüística, celebrada en setiembre de 2011 en Brasil. Son ideas, no obstante, que en muchos países van contra la corriente implantada por las políticas educativas (Civil y Planas, 2011), que en general son un obstáculo al aprendizaje matemático del alumno cuya lengua dominante no es oficial en la enseñanza. Moschkovich (2006) ha probado que el alumno se siente más confiado si se le permite explicar sus razonamientos recurriendo a su L1. Además, esta autora que ha probado que el uso de la L1 tiene un efecto positivo en la adquisición de autonomía durante la actividad matemática. Esta opción facilita la tarea del profesor, que puede contar en mayor medida con la iniciativa de los alumnos y la colaboración entre ellos. Incluso cuando el profesor no conoce la L1 de los alumnos y por paradójico que parezca, debe promoverse su uso, que será más efectivo si dos o más alumnos comparten la misma L1 y se incorpora el trabajo en grupos como dinámica de clase.

Ahora bien, el uso flexible de las lenguas no garantiza la efectividad de la comunicación en los procesos de enseñanza y aprendizaje de las matemáticas. Conviene seguir avanzando en la habilidad de usar las lenguas comunicativamente, esto es, creando un espacio para la negociación de significados y la resolución de problemas, ya sean relativos a contenidos de la enseñanza o a obstáculos inherentes a la comprensión de términos en una u otra lengua. En particular, el uso flexible de las lenguas se debe orientar al desarrollo de una comunicación efectiva. En nuestro estudio, hay elementos lingüísticos como la función del punto multiplicativo invisible en $x y z$ o la no presencia de la constante multiplicativa 1 en $100 x+10 y+z$, o bien el significado del término quiteña en una interacción donde otros alumnos no lo reconocen. Pero los obstáculos asociados a estas dificultades lingüísticas son educativamente relevantes cuando devienen problemas comunicativos. La comprensión de la L1, de la L2, del lenguaje algebraico... implica la comprensión de contenidos lingüísticos en relación estrecha con la función pragmática de lo que se expresa en el entorno comunicativo.

Cuando se compara $x y z$ y $100 x+10 y+z$, no hay interrupción en la comunicación, incluso a pesar de que Eduard no parece entender lo explicado por Amelia sobre su vivencia "quiteña". No hay, pues, auténticas rupturas lingüísticas que impidan la comunicación, sino más bien una dificultad por comprender la significatividad del mensaje en la discusión de una expresión algebraica donde no se contempla la representación física con material multibase. Tampoco hay interrupción en la comunicación cuando Roberto inventa la palabra batet, aunque no se incorpora la función pragmática implícita en el juego con catet y batet. Para este ejemplo, la 
comunicación matemática no llega a ser efectiva al no clarificarse el sentido atribuido al juego de palabras en todo su alcance. No falla la comprensión de la L1 ni de la L2, sino la de un juego que acompaña el proceso de aprendizaje del lenguaje algebraico, sobre el que ningún compañero de grupo se pronuncia. Sí hay interrupciones en la comunicación con repercusiones en el aprendizaje para otros momentos y casos. Por ejemplo cuando Silvia finaliza una discusión matemática con "Mira, es igual, así no acabaremos nunca".

Los resultados presentados deben interpretarse como inacabados, en espera de los datos y la profundización conceptual asociados a la segunda etapa del proyecto. No quiero acabar sin poner de relieve la red profesional que sustenta la investigación. El proyecto está siendo posible gracias a la colaboración con dos expertas en el ámbito, M. Civil y M. Setati, cuyos estudios se han realizado en países con una gran tradición científica en educación matemática y diversidad lingüística. Esta colaboración ha facilitado el acceso a fondos documentales del Centro CEMELA en Tucson y del Centro MARANG en Johannesburgo. Han sido necesarias estancias en ambos lugares y acciones de delimitación del proyecto (Chronaki, Setati, Planas, \& Civil, 2010). El gran reto sigue siendo afianzar esta línea de estudio en el contexto español (Planas, 2010, 2012), con estudiantes de doctorado que asuman esta temática e investigadores que se unan a un equipo con afán de crecimiento (Reverter, 2012). Además de mostrar resultados de la primera etapa del proyecto, otra motivación para escribir el artículo ha sido la de convencer sobre el aprendiz de matemáticas bilingüe como objeto sólido de estudio. Conviene ampliar el conocimiento en el área con el análisis del alumno bilingüe en entornos de iniciación al lenguaje algebraico, de manipulación de nociones geométricas, de uso de procesos estadísticos, etc. Para ello, todos los esfuerzos e iniciativas son pocos.

Agradecimientos. Proyecto EDU2009-07113 y EDU2012-31464, Ministerio de Economía y Competitividad. Proyecto ARFI2009-1-00052, Agència de Gestió d'Ajuts Universitaris i de Recerca. Proyecto C2010-10428-180881, Fundació Propedagògic y UAB.

\section{Referencias}

Chronaki, A., Setati, M., Planas, N., \& Civil, M. (2010). Same question different countries: Use of multiple languages in mathematics learning and teaching. En C. Morgan, U. Gellert, \& E. Jablonka (Eds.), Proceedings of the 6th International Conference on Mathematics Education and Society (Vol. 1, pp. 73-77). Berlín, Alemania: MES \& Free University of Berlin.

Civil, M. (2008). When the home language is different from the school language: Implications for equity in mathematics education. Comunicación presentada en el TSG 31 'Language and Communication in Mathematics Education', XI International Congress of Mathematics Education (ICME), Monterrey, México.

Civil, M. (2009). Mathematics education, language, and culture: Ponderings from a different geographic context. En R. Hunter, B. Bicknell, \& T. Burgess (Eds.), Crossing divides. Proceedings of the 32nd Annual Conference of the Mathematics Education Research Group of Australasia (Vol. 1, pp. 129-136). Palmerston North, Nueva Zelanda: MERGA.

Civil, M., \& Planas, N. (2011). Language policy and the teaching and learning of mathematics. En M. Setati, T. Nkambule, \& L. Goosen (Eds.), Proceedings of the ICMI Study 21 
Conference, Mathematics Education and Language Diversity (pp. 38-45). Sao Paulo, Brasil: ICMI.

Civil, M., \& Planas, N. (2012). Whose language is it? Reflections on mathematics education and language diversity from two contexts. In S. Mukhopadhyay \& W.-H. Roth (Eds.), Alternative forms of knowing (in) mathematics: Celebrations of diversity of mathematical practices (pp. 71-89). Rotterdam, Holanda: Sense Publishers.

Clarkson, P. C. (2006). Australian Vietnamese students learning mathematics: High ability bilinguals and their use of their languages. Educational Studies in Mathematics, 64(2), $191-215$.

Cummins, J. (2000). Language, power, and pedagogy: Bilingual children in the crossfire. Clevedon, Reino Unido: Multilingual Matters.

Drouhard, J. P., \& Teppo, A. (2004). Symbols and language. En K. Stacey, H. Chick, \& M. Kendal (Eds.), The future of the teaching and learning of algebra-The 12th ICMI Study (pp. 225-264). Nueva York, Estados Unidos: Springer.

Filloy, E., Puig, L., \& Rojano, T. (2008). El estudio teórico local del desarrollo de competencias algebraicas. Enseñanza de las Ciencias, 26(3), 327-342.

Font, V., \& Contreras, Á. (2008). The problem of the particular and its relation to the general in mathematics education. Educational Studies in Mathematics, 69, 33-52.

Glaser, B. G. (1969). The constant comparative method of qualitative analysis. En G. J. McCall, \& J. L. Simmons (Eds.), Issues in participant observation: A text and reader (pp. 216-228). Reading, Reino Unido: Addison-Wesley.

Goizueta, M., \& Planas, N. (2013). El papel del contexto en la identificación de argumentaciones matemáticas por un grupo de profesores. PNA, 7(4), 153-168.

INE-Instituto Nacional de Estadística (2011). Cifras de población y censos demográficos 2011. Recuperado de: http://www.ine.es/inebmenu/mnu_cifraspob.htm.

Kazima, M. (2006). Malawian students' meanings for probability vocabulary. Educational Studies in Mathematics, 64(2), 169-189.

Lins, R., \& Kaput, J. J. (2004). The early development of algebraic reasoning: The current state of the field. En K. Stacey, H. Chick, \& M. Kendal (Eds.), The future of the teaching and learning of algebra -The 12 $2^{\text {th }}$ ICMI Study (pp. 45-70). Nueva York, Estados Unidos: Springer.

MacGregor, M., \& Price, E. (1999). An exploration of aspects of language proficiency and algebra learning. Journal for Research in Mathematics Education, 30(4), 449-467.

Moschkovich, J. (2006). Using two languages when learning mathematics. Educational Studies in Mathematics, 64(2), 121-144.

Palarea, M. (1999). La adquisición del lenguaje algebraico: Reflexiones de una investigación. Números -Revista de Didáctica de las Matemáticas, 40, 3-28.

Parvanehnezhad, Z., \& Clarkson, P. C. (2008). Iranian bilingual students' use of language switching when solving mathematical problems. Mathematics Education Research Journal, 20(1), 51-80.

Pimm, D. (1987). Speaking mathematically: Communication in mathematics classrooms. Londres, Reino Unido: Routledge \& Kegan Paul.

Planas, N. (2010). Las teorías socioculturales en la investigación en educación matemática: datos bibliométricos y reflexiones. En M. M. Moreno, A. Estrada, J. Carrillo, \& T. Sierra (Eds.), Actas del XIV Simposio de la Sociedad Española de Investigación en Educación Matemática (pp. 163-198). Lleida, España: SEIEM. 
Planas, N. (2012). Hacia la recopilación de resultados sólidos en el área de educación matemática. En N. Planas (Ed.), Educación matemática: Teoría, crítica y práctica (pp. 11-26). Barcelona, España: Graó.

Planas, N. (2012). Heteroglossia and "orchestration" in multilingual mathematics classrooms. En H. Forgasz, \& F. Rivera (Eds.), Advances in Mathematics Education. Toward equity: gender, culture, and diversity (pp. 333-338). Nueva York, Estados Unidos: Springer

Planas, N., \& Civil, M. (2009). Working with mathematics teachers and immigrant students: an empowerment perspective. Journal of Mathematics Teacher Education, 12(6), 391-409.

Planas, N., \& Civil, M. (2010). El aprendizaje matemático de alumnos bilingües en Barcelona y Tucson. Quadrante -Revista Teorica e de Investigaçao, 29(1), 5-28.

Planas, N., \& Setati, M. (2009). Bilingual students using their languages in their learning of mathematics. Mathematics Education Research Journal, 21(3), 36-59.

Reverter, f. (2012). Mathematical learning and language use: Perspectives from bilingual students in a context of problem solving. Trabajo de Tesis Doctoral. Bellaterra, España: Universidad Autónoma de Barcelona.

Setati, M. (2005). Teaching mathematics in a primary multilingual classroom. Journal for Research in Mathematics Education, 36(5), 447-466.

Setati, M., \& Planas, N. (2012). Mathematics education across two different language contexts: a political perspective. En O. Skovsmose, \& B. Greer (Eds.), Opening the cage: Critique and politics of mathematics education (pp. 167-186). Rotterdam, Holanda: Sense Publishers.

Socas, M. M. (2002). La organización de los sistemas numéricos desde su estructura decimal: Algunas expresiones ambiguas. Números -Revista de Didáctica de las Matemáticas, 50, 19-34.

Socas, M. M. (2008). Dificultades y errores en el aprendizaje de las matemáticas. Análisis desde el enfoque lógico semiótico. En M. Camacho, P. Flores, \& M. P. Bolea (Eds.), Actas del XI Simposio de la Sociedad Española de Investigación en Educación Matemática (pp. 19-52). La Laguna, España: SEIEM.

Socas, M. M., Camacho, M., Palarea, M., \& Hernández, J. (1999). Iniciación al álgebra. Madrid, España: Síntesis.

Wittgenstein, L. (1953). Philosophische Untersuchungen. Oxford, Reino Unido: Basil Blackwell (consultada la traducción de 1988 por A. García \& U. Moulines, Investigaciones filosóficas. Barcelona, Crítica).

\section{Referencia a la autora}

Núria Planas, Universitat Autònoma de Barcelona (España).nuria.planas@uab.cat 


\title{
Initiation to the algebraic language in multilingual classrooms: contributions from an ongoing project
}

\author{
Núria Planas, Universitat Autònoma de Barcelona (Barcelona)
}

A vast body of literature in the area draws on the basic understanding of the relationship between attention to language diversity in multilingual classrooms and opportunities for mathematical learning. In the last decade investigations on such relationship have been intensified in the context of Catalonian secondary schools with classrooms in which more than one language is used in the teaching and learning of mathematics. In this article, the focus is on the analysis of classroom data as a way to identify both learning opportunities and difficulties that appear related to experiences of Catalan and Spanish bilingualism for the specific domain of algebra. The approach to the research with students is grounded on constant comparative methods inspired by Grounded Theory, along with the progressive development of emerging themes.

The article begins with a brief discussion of the theoretical notions of language role and use, followed by comments on the idea of becoming a bilingual learner of algebra and its language. All these considerations are to be interpreted in the political context of Catalonia, an autonomous region in North Eastern Spain where Catalan is the official language of instruction. Two cases of students, Roberto and Amelia, are then partially illustrated to better understand the phenomenon of students learning the language of algebra in a classroom with a language that is not their dominant home language. The two students speak Spanish at home, were born in the Republic of Ecuador, and have similar working-class backgrounds. Their parents did not complete high school, have limited Catalan proficiency, and immigrated to Catalonia for work reasons.

Results on learning opportunities and difficulties -for a total of seven cases of student- draw on observations of several mathematics lessons in secondary classrooms with Latin American Spanish and Catalan bilingual students. The lessons were planned so that the students spent most of the time working in linguistically homogeneous small groups determined in terms of the students' dominant language. There was also a whole class discussion at the end of the session when the students got a chance to share their different approaches to the task. In this didactical environment, it is explained the case of Roberto who uses geometric reasoning to model an algebraic expression, but takes a linguistic content to wrongly provide a guarantee to an argument. The case of Amelia shows how well another student downplays the differences between two algebraic expressions, but has difficulty making sense of the algebraic language due to an imprecise translation. Two emerging themes are detailed with classroom transcripts for each case.

On the one hand, it is argued that Roberto and Amelia overcome language constraints to go on with their mathematical learning in classrooms in which the language of teaching is not their dominant language. On the other, it is also argued that these same students face several difficulties in their learning due to the complex interaction between the ordinary languages -Spanish and Catalan- and the language of algebra. To a large extent, the investigation points to the fact of learning mathematics in a language other than the student's as not having an impact that is necessarily adverse or beneficial. The amount of results in this direction in the context of Catalonia and in other parts of the world is significant. However, the study of how the learning of mathematics is influenced by the practical orchestration of language diversity still deserves more work. 